\title{
Multi-agent Systems and Voting: How Similar Are Voting Procedures
}

\author{
Janusz Kacprzyk ${ }^{1,4(\otimes)}\left(\mathbb{D}\right.$, José M. Merigó ${ }^{2}$, Hannu Nurmi $^{3}$,
} and Sławomir Zadrożny ${ }^{1,4}{ }_{(\mathbb{D}}$

1 Systems Research Institute, Polish Academy of Sciences, ul. Newelska 6, 01-447 Warsaw, Poland kacprzyk@ibspan.waw.pl

2 Department of Management Control and Information Systems, University of Chile, Av. Diagonal Paraguay 257, 8330015 Santiago, Chile jmerigo@fen.uchile.cl

3 Department of Political Science, University of Turku, 20014 Turku, Finland hnurmi@utu.fi

${ }^{4}$ Warsaw School of Information Technology (WIT), 01-447 Warsaw, Poland

\begin{abstract}
We consider the problem of the evaluation of similarity of voting procedures which are crucial in voting, social choice and related fields. We extend our approach proposed in our former works and compare the voting procedures against some well established and intuitively appealing criteria, and using the number of criteria satisfied as a point of departure for analysis. We also indicate potential of this approach for extending the setting to a fuzzy setting in which the criteria can be satisfied to a degree, and to include a distance based analysis. A possibility to use elements of computational social choice is also indicated.
\end{abstract}

Keywords: Voting $\cdot$ Social choice $\cdot$ Voting procedure $\cdot$ Similarity $\cdot$ Binary pattern

\section{Introduction}

This paper is basically concerned with some aspects of a highly advocated trend of considering voting theory to be an important part of the broadly perceived area of multiagent systems (cf. Endriss [4], Pitt, Kamara, Sergot and Artikis [29], Dodevska [3], to just cite a few). In many multiagent systems we need a mechanism that can be used by the agents to make collective decisions, and an effective and efficient way of doing this can be by voting, that is by employing a voting procedure. There are very many voting procedures and there has been much research in this area, both related to theoretical analyses and development of voting protocols or procedures. Voting procedures are getting more complex, notably in view of a rapidly increasing importance of all kind of voting in large (maybe huge) sets of agents, for instance because of a proliferation of e-voting, and a rapidly increasing use of computers for this purpose. There is therefore 
an urgent need to use in the context of voting - which has traditionally been discussed within economics, decision theory or political science - new concepts, and tools and techniques of computer science and numerical analyses. This is basically offered by the new and rapidly developing area of computational social choice (cf. the handbook Brandt, Conitzer, Endriss, Lang and Procaccia [2]).

Multiagent systems are composed of agents, real or virtual entities exemplified by people (individuals, groups or organizations), software, etc. The agents act, interact, cooperate, collaborate, exchange information, etc. In multiagent systems decision processes, including voting, are usually highly uncertain and changeable (dynamic), and proceed in a distributed way, electronic voting is often advocated. In general, the winning option should be in line with opinions or preferences of as many agents as possible. Preferences, which are also an important topic in artificial intelligence (AI), are often used in multiagent models though it is not easy to elicit and deal with multiagent preferences.

We propose here to consider one of interesting problems in voting, both in the traditional social choice setting and a new multiagent setting, which can be stated as follows: there is a multitude of possible voting procedures proposed in the literature and it would be expedient to have a tool to determine if and to what extent they are similar to each other. This could help us, for instance, to use instead of a good but computationally demanding voting procedure a computationally simpler one if it is sufficiently similar to the former one.

In this paper we will discuss the similarity meant as how many requirements (conditions) which are usually assumed in voting are fulfilled jointly by a particular pair of voting procedures, and then to consider as similar those procedures which have similar values. We will use here the idea of our former, more qualitative approach to the comparison of voting procedures, cf. Kacprzyk, Nurmi and Zadrożny [11], and a more qualitative one by Fedrizzi, Kacprzyk and Nurmi [6]. It should be noted that an intuitively justified comparison of voting procedure might be with respect to the results obtained. This might however be too much related to a particular voting problem considered, and not general enough. One can argue, as we do, that the satisfaction (or not) of some important and widely accepted requirements for the voting procedure is possibly related to which solutions they can yield, that is, such a requirement related analysis can be implicitly equivalent to a result related analysis.

Moreover, in this paper we extend the above proposal by a new, fuzzy logic based one in which a "soft" measure of the number of requirements jointly satisfied is used which can be exemplified by a degree to which "about $n$, many, most, etc. requirements are fulfilled". This can be extended to the case of to which degree the requirements for the voting procedures are satisfied but this will not be considered here as it is not obvious and needs a deeper analysis of what social choice theorists and practitioners think about the intensity of satisfaction.

In our setting there are $n, n \geq 2$ individuals who present their testimonies over the set of $m, m \geq 2$, options. These testimonies can be, for instance, individual preference relations, orderings over the set of options, etc. In our approach we 
focus in principle on social choice functions, a class of social choice procedures that select a single social outcome, i.e. an option that best reflects the opinions of the individuals. Voting procedures are here perhaps the best known and most intuitively appealing examples. A voting procedure is meant to determine the winner of an election as a function of the votes cast by the voters.

Of a major concern in voting theory is the extent to which voting procedures satisfy some plausible and reasonable axioms, and more foundational results are of a rather negative type, that is, their essence is that plausible and intuitively obvious assumptions are usually incompatible, cf. the Arrow impossibility theorem. For more information, cf. Kelly [15], Nurmi [21], Riker [33], etc.

Much less attention has been paid to the problem of how similar/dissimilar are the voting procedures the number of which is very high. Except for a foundational reference book by Nurmi [21], and a new book by Teixeira de Almeida, Costa Morais and Nurmi [40], one can cite here: Elkind, Faliszewski and Slinko [5], McCabe-Dansted and Slinko [17], Richelson [32], etc.

In this paper we deal with this problem. We take into account a set of popular and well established criteria against which the voting procedures are usually evaluated. To reduce the size of this set, and the size of the set of the voting procedures, we use first the idea of Fedrizzi, Kacprzyk and Nurmi [6] in which these sets are reduced using a qualitative type analysis based on elements of Pawlak's [27], Pawlak and Skowron [28] rough sets theory to obtain the most specific non-redundant characterization of the particular voting procedures with respect to the criteria assumed.

Then, using this reduced representation, we consider the problem of how similar/dissimilar the particular voting procedures are, that is, of how to measure the degree of their similarity/dissimilarity. Our measure is derived from the number of criteria satisfied by a particular procedure, and in our view two voting procedures are similar if they jointly satisfy a similar number of criteria as proposed by Kacprzyk et al. [11]. As mentioned, this could be a good indicator of possibly similar results in terms of functioning, i.e. voting results yielded.

Next, we propose to extend this simple measure by using a fuzzy linguistic quantifier based aggregation to obtain the degree of satisfaction of, e.g., a few, many, most, much more than a half, etc. criteria. We also mention a possibility of using for the above fuzzy linguistic quantifier based aggregation the OWA operators, notably their recent extensions, cf. Kacprzyk, Yager and Merigó [12].

\section{A Comparison of Voting Procedures}

We are concerned with social choice functions which may be, for our purposes, equated with voting procedures. The literature on social choice is very rich, and a multitude of various social choice functions (voting procedures) has been proposed which can be simple and sophisticated, intuitively appealing and not, widely employed and not, etc. and there are little or no indications as to which one to use in a particular problem. For information on the comparison and evaluation of voting procedures, cf. the classic sources, e.g., Richelson [32], 
Straffin [37], Nurmi [21]; the recent book by Teixeira de Almeida et al., Costa Morais and Nurmi [40] provides much information on new approaches too.

In our context a simple and intuitive approach for the comparison of voting procedures using rough sets as a point of departure, has been proposed by Fedrizzi, Kacprzyk and Nurmi [6]. For a lack of space we will not present it here and refer the reader to that paper. We will just use as the point of departure the reduced problem representation obtained by using the rough sets.

We consider here the following 13 popular voting procedures:

1. Amendment: an option is proposed as a preliminary solution and then motions may be submitted to change it with another option; if such a motion gets required support then the proposed option is considered as a preliminary solution; if there are no more motions then the final vote for the current preliminary option is carried out,

2. Copeland: selects the option for which the number of times it beats other options minus the number of times it looses to other options in pairwise comparisons is the highest,

3. Dodgson: each voter provides a rank ordered list of all options, from the best to worst, and the option wins for which we need to perform the minimum number of pairwise swaps (summed over all candidate options) before they become a Condorcet winner,

4. Schwartz: selects the set of options over which the collective preferences are cyclic and the entire cycle is preferred over the other options; when a Condorcet winner exists this is the single element in such a set otherwise there may be many options,

5. Max-min: selects the option for which the greatest pairwise score for another option against it is the least one of score among all options,

6. Plurality: each voter selects one option (or none if abstains), and the options with the most selection votes win,

7. Borda: each voter provides a linear ordering of the options to which the socalled Borda score is assigned: in case of $n$ candidates, $n-1$ points is given to the first ranked option, $n-2$ to the second ranked, etc., these numbers are added up for the options over all orderings which yields the Borda count, and the option(s) with the highest Borda count wins.

8. Approval: each voter selects (approves) a subset of the candidate options and the option(s) with the most votes is/are the winner(s).

9. Black: selects the Condorcet winner, i.e. an option that beats or ties all other options in pairwise comparisons, when one exists or, otherwise, the Borda count winner (as described above),

10. Runoff: plurality vote is used first to find the top two options (or more if there are ties), and then there is a runoff between these options with the one with the most votes to win.

11. Nanson: the Borda count is used, at each step dropping the candidate with the smallest score (majority),

12. Hare: the ballots are linear orders over the set of options, and repeatedly the options are deleted which receive the lowest number of first places in the votes, and the option(s) that remain(s) are the winner(s), 
13. Coombs: each voter rank orders all of the options, and if one option is ranked first (among non-eliminated options) by an absolute majority of the voters, then this is the winner, otherwise, the option which is ranked last by a plurality of the voters is eliminated, and this is repeated.

Notice that these voting procedures are well known and popular but, clearly, are just examples of a multitude of possible procedures known in the literature and even employed (cf. the book by Teixeira de Almeida et al. [40]). For example, some recent promising procedures are not used in this paper as Schulze's method [35], Tideman's ranked pairs method [38], and many other ones.

These voting procedures used are based on highly reasonable, desirable and intuitively appealing properties but it is difficult to say if and how similar or different they are. Such an analysis of similarity/dissimilarity can proceed by comparing the voting procedures against some well founded and reasonable criteria (requirements). A multitude of various criteria are possible, and no voting procedure will satisfy all of them. The comparison of voting procedures is therefore a non-trivial task and is to a large extent subjective. We will try to use some formal tools to make it more objective.

The satisfaction of the following criteria is often advocated (cf. Nurmi [21]):

1. Majority winner criterion: if there exists a majority (at least 50\%) of voters who rank a single option at the top of the ranking, higher than all other options, then this option should win,

2. Mutual majority criterion: if there exists a majority of voters ranking a group of options higher than all other options, one of the options from that group should win.

3. Majority loser criterion: if a majority of voters prefers every other option over a given one, the latter option should not win,

4. Monotonicity criterion: it is impossible to make a winning option lose by ranking it higher, or to cause a losing option to win by ranking it lower,

5. Consistency criterion: if the electorate is divided in two groups and an option wins in both groups, then it should win in general,

6. Weak Pareto criterion: whenever all voters rank an option higher than another option, the latter option should never be chosen,

7. Participation criterion: it should always be better to vote honestly than not to vote at all,

8. Condorcet winner criterion: if an option beats every other option in pairwise comparisons, then it should always win,

9. Condorcet loser criterion: if an option loses to every other option in pairwise comparisons, it should always loose,

10. Independence of irrelevant alternatives: if an option is added or removed, the relative rankings of the remaining options should remain the same,

11. Independence of clones: the outcome of voting should be the same if we add options identical to the existing ones (clones),

12. Reversal symmetry: if individual preferences of each voter are inverted, the original winner should never win, 
13. Heritage criterion: if an option is chosen from the entire set of options using a particular voting procedure, then it should also be chosen from all subsets of the set of options (to which it belongs) using the same voting procedure and under the same preferences.

14. Polynomial time: it should be possible to find the winner in polynomial time with respect to the number of options and voters.

In general, a criterion can be said to be "stronger" (more widely adopted/adhered to) than another one when it is satisfied by more voting procedures. This will be of relevance for our consideration.

For clarity and simplicity of presentation and interpretation, we will only use the following 7 "strong" criteria (the letters A, .., G correspond to the labels of columns in the tables to be shown), which can be claimed to be especially important (cf. Fedrizzi, Kacprzyk and Nurmi [6]), in the analysis and comparison of voting procedures: A - Condorcet winner, $\mathrm{B}$ - Condorcet loser, $\mathrm{C}$ - majority winner, D - monotonicity, E - weak Pareto winner, F - consistency, and G heritage, and a similar analysis can be extended to all 13 criteria listed before, as well as many other ones which can be found in the literature.

In the tables showing results of the subsequent steps of our approach, the rows will correspond to the 13 voting procedures analyzed in the paper: Amendment, Copeland, Dodgson, Schwartz, Max-min, Plurality, Borda, Approval, Black, Runoff, Nanson, Hare, and Coombs.

The columns correspond to 7 above mentioned criteria: Condorcet winner, Condorcet loser, majority winner, monotonicity, weak Pareto winner, consistency, and heritage.

The point of departure is presented in Table 1 which shows which voting procedure satisfies which criterion: "0" stands for "does not satisfy", and "1" stands for "satisfies".

It should be noticed that the data set given in Table 1 can be directly used for the comparison of the 13 voting procedures considered with respect to the 7 criteria assumed. Basically, such a comparison can be accomplished by comparing the consecutive pairs of the binary rows corresponding to the voting procedures with each other using some methods for the determination of similarity and dissimilarity (cf. Kacprzyk et al. [11]). However, this would not provide any deeper insight into the differences between the voting procedures as the comparison would concern just particular voting procedures and not their more or less homogeneous classes. This problem is closely related to Kacprzyk and Zadrożny's $[13,14]$ OWA operator based approach to the classification of voting procedures into a number of more general classes that are related, first, to the order in which the aggregation via an OWA operator proceeds and, second, to specific sets of weights of the respective OWA operators. In this paper we use another way of comparing the voting procedures based on an analysis of how many criteria are jointly fulfilled, and on the related distance between the voting procedures, and then on some structural analyses using human consistent natural language summaries. 
Table 1. Satisfaction of 7 criteria by 13 voting procedures

\begin{tabular}{l|l|l|l|l|l|l|l}
\hline \multirow{2}{*}{ Voting procedure } & \multicolumn{6}{|c}{ Criteria } \\
\cline { 2 - 9 } & A & B & C & D & E & F & G \\
\hline Amendment & 1 & 1 & 1 & 1 & 0 & 0 & 0 \\
\hline Copeland & 1 & 1 & 1 & 1 & 1 & 0 & 0 \\
\hline Dodgson & 1 & 0 & 1 & 0 & 1 & 0 & 0 \\
\hline Schwartz & 1 & 1 & 1 & 1 & 0 & 0 & 0 \\
\hline Max-min & 1 & 0 & 1 & 1 & 1 & 0 & 0 \\
\hline Plurality & 0 & 0 & 1 & 1 & 1 & 1 & 0 \\
\hline Borda & 0 & 1 & 0 & 1 & 1 & 1 & 0 \\
\hline Approval & 0 & 0 & 0 & 1 & 0 & 1 & 1 \\
\hline Black & 1 & 1 & 1 & 1 & 1 & 0 & 0 \\
\hline Runoff & 0 & 1 & 1 & 0 & 1 & 0 & 0 \\
\hline Nanson & 1 & 1 & 1 & 0 & 1 & 0 & 0 \\
\hline Hare & 0 & 1 & 1 & 0 & 1 & 0 & 0 \\
\hline Coombs & 0 & 1 & 1 & 0 & 1 & 0 & 0 \\
\hline
\end{tabular}

Table 2. Satisfaction of 7 criteria by a reduced number (9 families) of voting procedures

\begin{tabular}{l|l|l|l|l|l|l|l}
\hline \multirow{2}{*}{ Voting procedure } & \multicolumn{6}{|c}{ Criteria } \\
\cline { 2 - 9 } & A & B & C & D & E & F & G \\
\hline Amendment & 1 & 1 & 1 & 1 & 0 & 0 & 0 \\
\hline Copeland & 1 & 1 & 1 & 1 & 1 & 0 & 0 \\
\hline Dodgson & 1 & 0 & 1 & 0 & 1 & 0 & 0 \\
\hline Max-min & 1 & 0 & 1 & 1 & 1 & 0 & 0 \\
\hline Plurality & 0 & 0 & 1 & 1 & 1 & 1 & 0 \\
\hline Borda & 0 & 1 & 0 & 1 & 1 & 1 & 0 \\
\hline Approval & 0 & 0 & 0 & 1 & 0 & 1 & 1 \\
\hline Runoff & 0 & 1 & 1 & 0 & 1 & 0 & 0 \\
\hline Nanson & 1 & 1 & 1 & 0 & 1 & 0 & 0 \\
\hline
\end{tabular}

The first step of the approach proposed in Fedrizzi et al. [6] and Kacprzyk et al. [11] is the simplification of the problem in the sense of the reduction of the number of voting procedures using elements of Pawlak's rough sets theory (cf. Pawlak [27], Pawlak and Skowron [28]). Basically, first, we merge those voting procedures which satisfy the same properties, i.e. under the set of criteria assumed they may be considered to be equivalent. We obtain therefore the following 9 voting procedures (Table 2): Amendment (which stands now for Amendment and Schwartz), Copeland (which stands now for Copeland and Black), Dodgson, Max-min, Plurality, Borda, Approval, Runoff (which stands now for Runoff, Hare and Coombs), and Nanson. These are equivalence classes of the indiscernibility relation which may be defined as usual, in line with the rough sets theory.

This step may be followed by another one aiming at reducing also the number of criteria. One may identify the so-called indispensable criteria (the core) which are meant as those whose omission will make at least one pair of voting procedures indistinguishable. That is, such criteria are necessary to differentiate between the voting procedures. Then, we finally obtain the reduced representation of the voting procedures versus the criteria as shown in Table 3 which expresses the most crucial properties or criteria of the voting procedures in the sense that the information it conveys would be sufficient to restore all information given in the source Table 1. For details we refer the reader to cf. Fedrizzi, Kacprzyk and Nurmi [6] and Kacprzyk, Nurmi and Zadrożny [11]. However, in this paper we will assume that only the first step is executed, i.e., the number of voting procedures is reduced but all the criteria are preserved. 
Table 3. Satisfaction of the criteria belonging to the core by the particular voting procedures

\begin{tabular}{l|l|l|l|l}
\hline \multirow{2}{*}{ Voting procedure } & \multicolumn{4}{|c}{ Criteria } \\
\cline { 2 - 5 } & A & B & D & E \\
\hline Amendment & 1 & 1 & 1 & 0 \\
\hline Copeland & 1 & 1 & 1 & 1 \\
\hline Dodgson & 1 & 0 & 0 & 1 \\
\hline Max-min & 1 & 0 & 1 & 1 \\
\hline Plurality & 0 & 0 & 1 & 1 \\
\hline Borda & 0 & 1 & 1 & 1 \\
\hline Approval & 0 & 0 & 1 & 0 \\
\hline Runoff & 0 & 1 & 0 & 1 \\
\hline Nanson & 1 & 1 & 0 & 1 \\
\hline
\end{tabular}

\section{Similarity and Distances Between Voting Procedures: An Indiscernibility Based Analysis}

We operate here on the characterization of the voting procedures shown in the Table 2. This will better serve the purpose of presenting a new approach to the comparison of voting procedures, and also provide a point of departure for further works in which similarity analyses will be performed on reduced representations.

For each pair of voting procedures, $(x, y) \in V^{2}$, where $V$ is the set of voting procedures (9 in our case, as in Table 2), and for each criterion $z, z \in Z$, where $Z$ is the set of criteria assumed ( 7 in our case, as in Table 1 ), we define the following function $v_{z}: V \times V \longrightarrow\{0,1\}$, such that

$$
v_{z}(x, y)=\left\{\begin{array}{l}
1 \text { if } x \text { and } y \text { take on the same values for criterion } z \\
0 \text { otherwise }
\end{array}\right.
$$

For example, for the data given in Table 1:

$$
\begin{aligned}
& v_{\mathrm{A}}(\text { Amendment }, \text { Copeland })=1 \\
& v_{\mathrm{E}}(\text { Amendment }, \text { Copeland })=0
\end{aligned}
$$

In the simplest way the agreement between two voting procedures, $x, y \in V$, denoted by $A(x, y), A: V \times V \longrightarrow\{0, \ldots$, card $Z\}$, can be defined in terms of $v_{z}(x, y)$ given by $(1)$ as follows:

$$
A(x, y)=\sum_{z \in Z} v_{z}(x, y)
$$

that is as the number of simultaneous satisfaction/dissatisfaction of the criteria. 
Therefore, we get the following matrix of agreements (cf. Table 4). In Table 4, the agreement between the same voting procedures does not matter, so that "-" is put, and since the agreement function is symmetric, we only define the upper half of the matrix.

Table 4. Values of agreements between the particular voting procedures due to (2)

\begin{tabular}{|c|c|c|c|c|c|c|c|c|c|}
\hline \multirow{2}{*}{$\begin{array}{l}\text { Voting } \\
\text { procedure }\end{array}$} & \multicolumn{9}{|c|}{ Voting procedure } \\
\hline & Amendment & Copeland & Dodgson & Max-min & Plurality & Borda & Approval & Runoff & Nanson \\
\hline Amendment & - & 6 & 4 & 5 & 3 & 3 & 1 & 4 & 5 \\
\hline Copeland & & - & 5 & 6 & 4 & 4 & 1 & 5 & 6 \\
\hline Dodgson & & & - & 6 & 4 & 2 & 2 & 5 & 6 \\
\hline Max-min & & & & - & 5 & 3 & 2 & 4 & 5 \\
\hline Plurality & & & & & - & 5 & 4 & 4 & 3 \\
\hline Borda & & & & & & - & 4 & 4 & 3 \\
\hline Approval & & & & & & & - & 1 & 0 \\
\hline Runoff & & & & & & & & - & 6 \\
\hline Nanson & & & & & & & & & - \\
\hline
\end{tabular}

It can be illustrative to present the results in the form of some summarizing statements. One can notice that the Copeland, Max-Min, Dodgson and Nanson form a group of voting procedures which are no more than two criteria away of each other. Quite closely related to that group are Runoff and Amendment. The so-called positional methods, that is, Plurality, Borda and Approval, seem to be rather far away from the rest of the procedures in terms of the number of criteria they differ by. This holds particularly for Approval.

It is easy to see that this indiscernibility analysis based on the sets of criteria satisfied jointly by pairs of voting procedures yields here sets of cardinality 0 , $1,2,3,4,5,6$. Of course, in practice it is usually not important if two voting procedures differ by 2 or 3,4 or 5 , or 5 or 6 criteria so that one can use here aggregated values, for instance, by merging these values, which would yield a more compact representation.

However, such a merging of numbers of criteria may be difficult because it needs a deep insight into how important the particular criteria are, and the satisfaction of which combination of them is relevant.

Moreover, it may often be convenient to use a natural language description of the similarity, for instance similar in terms of: a low, medium or high, a few and many, about $n$, etc. number of criteria satisfied.

Technically, this can easily be done by using tools and techniques of fuzzy logic, to be more specific the well known fuzzy logic based calculus of linguistically quantified propositions by Zadeh.

The method presented in this section, and the results obtained, which is based on some indiscernibility analyses, may be viewed to be somewhat qualitative. To proceed to a more quantitative analysis, we can use the normalized 


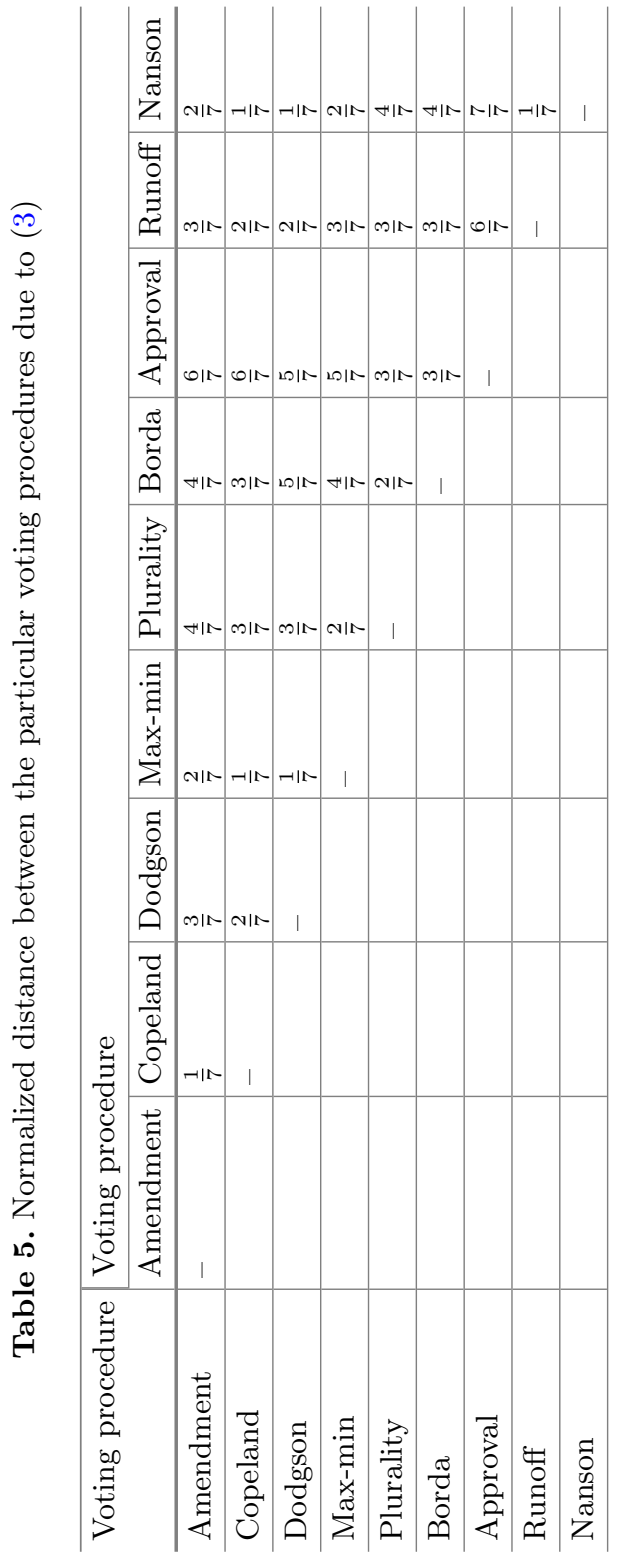


distance between two voting procedures $x, y \in V$ which can be defined in a straightforward way as

$$
D(x, y)=1-\frac{A(x, y)}{\operatorname{card} Z}
$$

where $A(x, y)$ is given by $(2)$ and card $Z$ is the number of criteria.

Therefore, using (3), we obtain the matrix of normalized distances between the voting procedures given by Table 5 .

Then, a distance based analysis can be performed along the lines of Kacprzyk, Nurmi and Zadrożny [11] but this is outside of the scope of this paper that is focused on an indiscernibility analysis.

\section{Concluding Remarks}

We have presented a new approach to the evaluation of similarity/dissimilarity of voting procedures. We followed the approach proposed in our former works and compared the voting procedures against some well established and intuitively appealing criteria, and using the number of criteria satisfied as a point of departure. We have indicated some further research directions, notably using elements of fuzzy logic to describe the delicacy of the comparison of voting procedures and also a possibility to extend the analysis to a distance based reasoning.

We hope that this work will help solve one of problems that exists in the use of voting procedures in multiagent systems (cf. Dodevska [3] or Endriss [4]). Moreover, for large scale voting problems which are more and more important in practice, computational social sciences can provide a rich set of tools and techniques which will help solve our problem (cf. Elkind, Faliszewski and Slink [5]).

\section{References}

1. Arrow, K.J., Sen, A.K., Suzumura, K. (eds.): Handbook of Social Choice and Welfare, 1st edn. Elsevier, Amsterdam (2002)

2. Brandt, F., Conitzer, V., Endriss, U., Lang, J., Procaccia, A.D. (eds.): Handbook of Computational Social Choice. Cambridge University Press, Cambridge (2016)

3. Dodevska, Z.: Voting in multi-agent systems. Tehnika-Menadžment 69(5), 724-740 (2019)

4. Endriss, U.: Social choice theory as a foundation for multiagent systems. In: Müller, J.P., Weyrich, M., Bazzan, A.L.C. (eds.) MATES 2014. LNCS (LNAI), vol. 8732, pp. 1-6. Springer, Cham (2014). https://doi.org/10.1007/978-3-319-11584-9_1

5. Elkind, E., Faliszewski, P., Slinko, A.: On the role of distances in defining voting rules. In: van der Hoek, W., Kaminka, G.A., Lespérance, Y., Luck, M., Sen, S. (eds.) Proceedings of 9th International Conference on Autonomous Agents and Multiagent Systems (AAMAS 2010), pp. 375-382 (2010)

6. Fedrizzi, M., Kacprzyk, J., Nurmi, H.: How different are social choice functions: a rough sets approach. Qual. Quant. 30, 87-99 (1996)

7. Gibbard, A.: Manipulation of voting schemes: a general result. Econometrica 41(4), 587-601 (1973) 
8. Kacprzyk, J.: Group decision making with a fuzzy majority. Fuzzy Sets Syst. 18, 105-118 (1986)

9. Kacprzyk, J., Fedrizzi, M.: A 'human consistent' degree of consensus based on fuzzy logic with linguistic quantifiers. Math. Soc. Sci. 18, 275-290 (1989)

10. Kacprzyk, J., Fedrizzi, M., Nurmi, H.: Group decision making and consensus under fuzzy preferences and fuzzy majority. Fuzzy Sets Syst. 49, 21-31 (1992)

11. Kacprzyk, J., Nurmi, H., Zadrożny, S.: Using similarity and dissimilarity measures of binary patterns for the comparison of voting procedures. In: Kacprzyk, J., Filev, D., Beliakov, G. (eds.) Granular, Soft and Fuzzy Approaches for Intelligent Systems. SFSC, vol. 344, pp. 141-169. Springer, Cham (2017). https://doi.org/10. 1007/978-3-319-40314-4_8

12. Kacprzyk, J., Yager, R.R., Merigó, J.M.: Towards human-centric aggregation via ordered weighted aggregation operators and linguistic data summaries: a new perspective on Zadeh's inspirations. IEEE Comput. Intell. Mag. 14(1), 16-30 (2019). https://doi.org/10.1109/MCI.2018.2881641

13. Kacprzyk, J., Zadrożny, S.: Towards a general and unified characterization of individual and collective choice functions under fuzzy and nonfuzzy preferences and majority via the ordered weighted average operators. Int. J. Intell. Syst. 24(1), 4-26 (2009)

14. Kacprzyk, J., Zadrożny, S.: Towards human consistent data driven decision support systems using verbalization of data mining results via linguistic data summaries. Bull. Pol. Acad. Sci.: Tech. Sci. 58(3), 359-370 (2010)

15. Kelly, J.S.: Arrow Impossibility Theorems. Academic Press, New York (1978)

16. Kelly, J.S.: Social Choice Theory. Springer, Berlin (1988). https://doi.org/10.1007/ 978-3-662-09925-4

17. McCabe-Dansted, J.C., Slinko, A.: Exploratory analysis of similarities between social choice rules. Group Decis. Negot. 15(1), 77-107 (2006)

18. Moulin, H.: The strategy of social choice. Advanced Textbooks in Economics. North-Holland, Amsterdam (1983)

19. Moulin, H.: Axioms of Cooperative Decision Making. Cambridge University Press, Cambridge (1991)

20. Merrill, S.: Making Multicandidate Elections More Democratic. Princeton University Press, Princeton (1988)

21. Nurmi, H.: Comparing Voting Systems. D. Reidel, Dordrecht (1987)

22. Nurmi, H.: Discrepancies in the outcomes resulting from different voting schemes. Theory Decis. 25, 193-208 (1988a)

23. Nurmi, H.: Inferential modes in applying social choice theory. In: Munier, B.R., Shakun, M.F. (eds.) Compromise, Negotiation and Group Decision. D. Reidel, Dordrecht (1988b)

24. Nurmi, H.: An assessment of voting system simulations. Publ. Choice 73, 459-487 (1992)

25. Nurmi, H., Kacprzyk, J.: On fuzzy tournaments and their solution concepts in group decision making. Eur. J. Oper. Res. 51, 223-232 (1991)

26. Nurmi, H., Kacprzyk, J., Fedrizzi, M.: Probabilistic, fuzzy and rough concepts in social choice. Eur. J. Oper. Res. 95, 264-277 (1996)

27. Pawlak, Z.: Rough Sets: Theoretical Aspects of Reasoning About Data. Kluwer, Dordrecht (1991)

28. Pawlak, Z., Skowron, A.: Rudiments of rough sets. Inf. Sci. 177(1), 3-27 (2007)

29. Pitt, J., Kamara, L., Sergot, M., Artikis, A.: Voting in multi-agent systems. Comput. J. 49(2), 156-170 (2006) 
30. Plott, C.R.: Axiomatic social choice theory: an overview and interpretation. Am. J. Polit. Sci. 20, 511-596 (1976)

31. Shoham, Y., Leyton-Brown, K.: Multiagent Systems: Algorithmic, Game Theoretic, and Logical Foundations. Cambridge University Press, Cambridge (2009)

32. Richelson, J.: A comparative analysis of social choice functions I, II, III: a summary. Behav. Sci. 24, 355 (1979)

33. Riker, W.H.: Liberalism Against Populism. W. H. Freeman, San Francisco (1982)

34. Satterthwaite, M.A.: Strategy-proofness and Arrow's conditions: existence and correspondence theorems for voting procedures and social welfare functions. J. Econ. Theory 10, 187-217 (1975)

35. Schulze, M.: A new monotonic, clone-independent, reversal symmetric, and Condorcet-consistent single-winner election method. Soc. Choice Welfare 36(2), 267-303 (2011)

36. Schwartz, T.: The Logic of Collective Choice. Columbia University Press, New York (1986)

37. Straffin, P.D.: Topics in the Theory of Voting. Birkhäuser, Boston (1980)

38. Tideman, N.T.: Collective Decisions and Voting: The Potential for Public Choice. Ashgate Publishing, Farnham (2006)

39. Yager, R.R., Kacprzyk, J., Beliakov, G. (eds.): Recent Developments in the Ordered Weighted Averaging Operators: Theory and Practice. Springer, Heidelberg (2011). https://doi.org/10.1007/978-3-642-17910-5

40. de Almeida, A.T., Morais, D.C., Nurmi, H.: Systems, Procedures and Voting Rules in Context. AGDN, vol. 9. Springer, Cham (2019). https://doi.org/10.1007/9783-030-30955-8 